\title{
On the general structure of giant low surface brightness galaxy Malin 2
}

\author{
A. S. Saburova ${ }^{1}$, A. V. Kasparova ${ }^{1}$, I. Yu. Katkov ${ }^{1}$, D. V. Bizyaev ${ }^{2,1}$ \\ and I. V. Chilingarian ${ }^{3,1}$ \\ ${ }^{1}$ Sternberg Astronomical Institute of Moscow State University, \\ Universitetskii pr. 13, 119992, Moscow, Russia \\ email: saburovaann@gmail.com \\ ${ }^{2}$ New Mexico State University and Apache Point Observatory, Sunspot, NM 88349, USA \\ ${ }^{3}$ Smithsonian Astrophysical Observatory, Harvard-Smithsonian Center for Astrophysics, 60 \\ Garden Street, Cambridge, MA 02138 USA
}

\begin{abstract}
We carried out the multicolor surface photometry in BVR and griz filters parallel with stellar kinematic measurements from the long slit spectra for the low surface brightness galaxy Malin 2. The use of the multicolor surface photometry as well as the available HI rotation curve allowed us to construct the mass distribution model of the galaxy. Photometrical and dynamical mass estimates agree with the dark halo mass fraction of about $70 \%$ within four disc radial scalelengths $(\sim 70 \mathrm{kpc})$. We used our dynamical model to obtain radial profiles of the equilibrium disc volume density and gas pressure in the galaxy midplane based on the available $\mathrm{HI}$ and $\mathrm{CO}$ data. The observed molecular gas fraction appears to be much higher than in the high surface brightness galaxies for a similar gas pressure.
\end{abstract}

Keywords. Galaxies: evolution, Galaxies: formation, Galaxies: individual: Malin 2.

We decomposed the HI rotation curve of Malin 2 obtained by Pickering et al. (1997) into four components: pseudoisothermal halo, exponential stellar disc, Sersic bulge and gaseous disc. We assumed that mass is following light (R-band) for the stellar disc and bulge radial profiles. The $M / L_{R}$ ratios of disc and bulge were calculated from the observed color profiles and stellar population models. The obtained photometrical model is close to the "maximum disc" model.

We calculated the equilibrium hydrostatic turbulent gas pressure using an equilibrium self-consistent model (see Kasparova 2012). We find that Malin 2 behaves in a different way than the normal spiral field galaxies. For all points along the radius the values of $\eta$ (molecular gas fraction) is significantly higher than the expected ones for a given $P$ in field galaxies.

A possible explanation is the long lifetime of molecular clouds in low density regions.

This work was supported by president grant of the Russian Federation MD-3288.2012.2.

\section{References}

Das, M., Boone, F., \& Viallefond, F. 2010, $A \mathscr{E} A, 523,63$

Pickering, T. E., Impey, C. D., van Gorkom, J. H., \& Bothun, G. D. 1997, AJ, 114, 1858

Kasparova, A. 2012 Astron. Lett., 38, 63

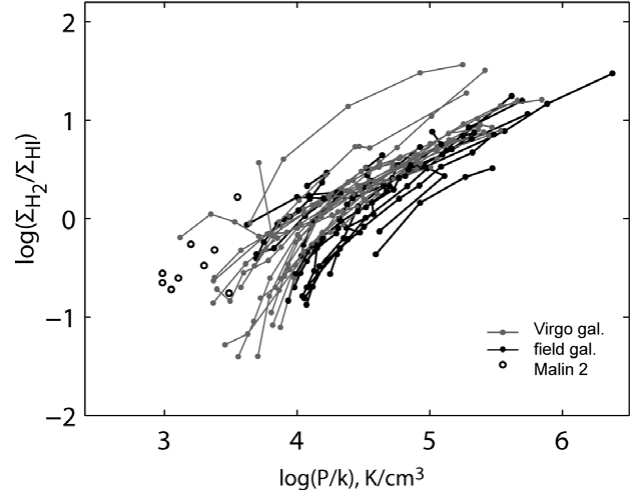

Figure 1. The molecular gas fraction $(\eta)$ versus the gas pressure. 